\title{
Influence of HVDC Transmission on DC Bias of AC Grid and Its Treatment
}

\author{
Ruifeng ZHAN ${ }^{\mathrm{a}}$, Yuying HU' ${ }^{\mathrm{a}}$, Fan $\mathrm{LI}^{\mathrm{b}}$, Zhou MIc and Lingpeng DONG ${ }^{\mathrm{d}, 1}$ \\ ${ }^{a}$ Huzhou Electric Power Design Institute Company Limited, Huzhou, China \\ b State Grid Huzhou Power Supply Company, Huzhou, China \\ ' State Grid Hangzhou Power Supply Company, Hangzhou, China \\ ${ }^{\mathrm{d}}$ Zhejiang University, Hangzhou, China
}

\begin{abstract}
With the rapid development of China's electric power industry, the high-voltage and long-distance direct current (DC) transmission effectively solved the problem of uneven power distribution. When the high voltage direct current transmission is in unipolar operation or bipolar asymmetric operation, part of the DC current will flow into the transformer winding through the grounded neutral point, which will cause the DC bias problem. This article used CDEGS software for modeling, and introduced the process of CDEGS software for DC bias simulation modeling. In this paper, the DC bias model of regional power grid is first established, based on the Zhejiang power grid topology and the test soil resistivity date. Then the DC bias currents of the transformers are calculated, and finally the corresponding treatment measures are proposed. According to the governance measures, this article adjusted the simulation model. The calculation results show that the treatment measures have good effects, which can provide an important reference for the future treatment of transformer DC bias.
\end{abstract}

Keywords. Electric power, transformer DC bias, DC bias model

\section{Introduction}

High voltage direct current (HVDC) transmission has developed rapidly in many countries due to its advantages of large transmission capacity, long transmission distance and low line loss [1-3]. However, when the HVDC is under maintenance or failure, it will implement unipolar operation or bipolar asymmetric operation. The DC current will flow back through the earth and flow into the transformers by the grounded neutral point, causing the transformers DC bias effect [4-8]. The DC biasing effect of the transformers will cause abnormal vibration, increase of harmonics, and malfunction of relay protection. In order to solve the problems, domestic and foreign scholars have carried out a lot of research, mainly focusing on two aspects: (1) the distribution of DC current in the AC power grid; (2) the harm of DC bias to power equipments and its treatment measures. This paper focuses on the DC bias problem of Baihetan-Zhejiang DC under construction. We collected the topological structure of Zhejiang Power Grid, tested the soil resistivity around the grounding pole, established the DC bias calculation model of Zhejiang Power Grid, and calculated the DC bias distribution current of the regional power grid, and finally proposed targeted treatment measures.

1 Corresponding Author, Lingpeng DONG, Zhejiang University, Hangzhou, China; Email: 21810015@zju.edu.cn. 


\section{DC Bias Calculation Model}

\subsection{Grid Topology Model}

The ground electrode of $\pm 800 \mathrm{kV}$ Baihetan-Zhejiang HVDC is located in Chen'an Village, Huzhou city. Due to the higher unit density and complex structure of Zhejiang Power Grid, it will have a major impact on the DC bias of Zhejiang Power Grid. Similarly, the Jinsu HVDC ground electrode around Huzhou City will also affect the substations in Zhejiang area. Therefore, it is necessary to comprehensively consider the DC bias effect of the two HVDC transmissions in the model $[9,10]$.

According to the classic grounding theory, when a large current is injected into the ground along with the grounding electrode, a spatial electric field will be formed in the ground, and the ground potential of the surrounding area will change. When the neutral point of the substation is grounded, the DC current will flow into the transformer by the neutral point and flows to the remote substations through the overhead lines connected to each other. Therefore, to calculate the distribution of DC current in transformers, two models of electric field and circuit must be considered at the same time.

There are multiple connection types for transformer, bus and overhead lines of the substation. According to different connection types, this paper equates the transformer to six basic forms, as shown in figure 1 . Where $R_{g}$ is the transformer grounding resistance, $R_{c}$ is the DC resistance of the autotransformer common winding, $R_{s}$ is the autotransformer series winding DC resistance, $R_{M}$ is the non-autotransformer medium voltage side winding resistance, $R_{H}$ is the non-autotransformer high voltage side winding, the subscript number is the main transformer numbering.

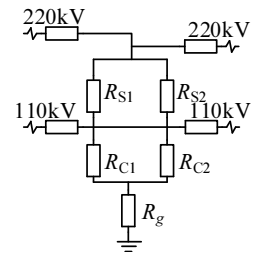

(a) Parallel operation of autotransformers

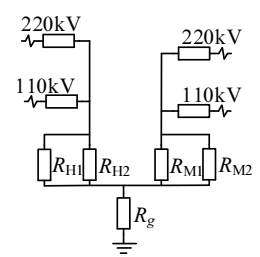

(d) Parallel operation of nonautotransformers

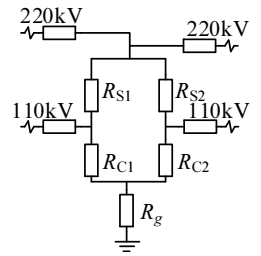

(b) High-voltage parallel and mediumvoltage separate operation of autotransformer

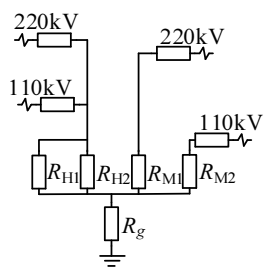

(e) High-voltage parallel and mediumvoltage separate of non-autotransformer

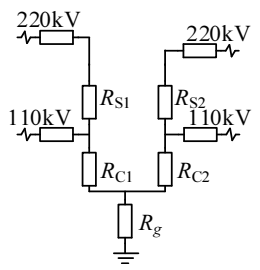

(c) Separate operation of autotransformer

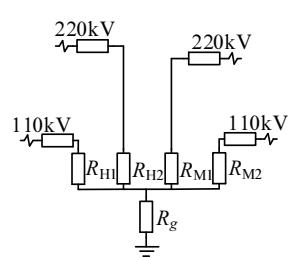

(f) Separate operation of nonautotransformers

Figure 1. Equivalent resistance network of different transformer connection methods.

In order to fully consider the interaction between the substations and the transmission lines, this paper takes the AC grid around the DC ground electrode as the research object, and establishes the DC bias simulation model of Zhejiang Power Grid. 
In the model, total number of substations is 1252 , including 5 substations of $1000 \mathrm{kV}$, 54 substations of $500 \mathrm{kV}, 238$ substations of $220 \mathrm{kV}$, and 955 substations of $110 \mathrm{kV}$. The total number of transmission lines is 1288 , including 9 lines of $1000 \mathrm{kV}, 53$ lines of $500 \mathrm{kV}$, and 347 lines of $220 \mathrm{kV}, 879$ lines of $110 \mathrm{kV}$, the model as shown in figure 2 . The straight line represents the transmission line, and the dot represents the substation.

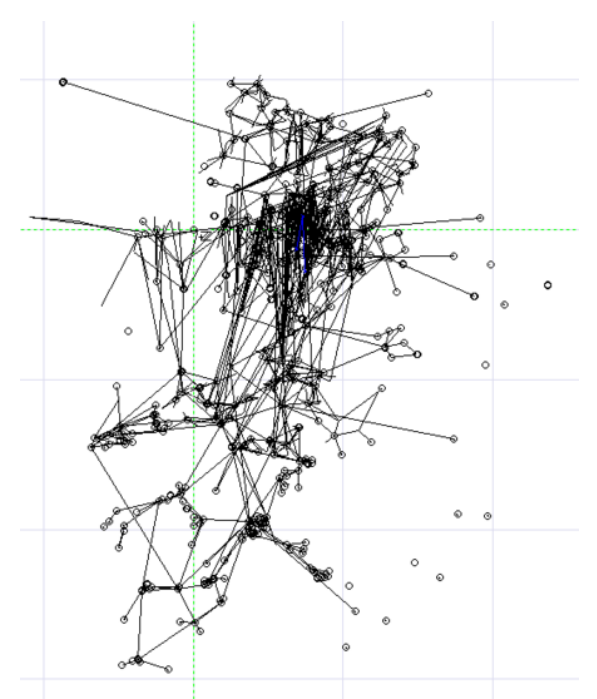

Figure 2. Simulation model of DC Bias in Zhejiang power grid.

\subsection{Soil Resistivity}

The soil model is a key parameter in the DC bias calculation. In order to obtain accurate soil parameters. The deep soil resistivity of 10 substations was detected, including Ji'an, Anshan Square, Jindingzi, Wandan Village, Huangzhi, Baique, Nanxun, Taijia, Yingxi and Heiqiaozi. The distribution of substations is shown in figure 3. The four-pole method and magnetotelluric sounding method are used to detect the soil resistivity near the DC ground electrode, and the detection depth is $10 \mathrm{~km}$.

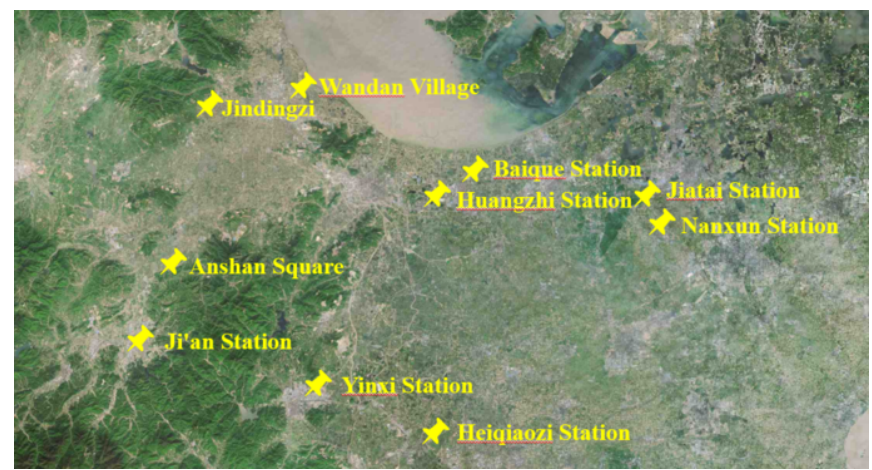

Figure 3. The distribution of substations.

Combining the single-point electrical sounding results of 10 substations, the resistivity contour maps of different depths are formed. When setting up the soil 
parameter model, the horizontal layered model is generally used, so this paper simplified the soil electrical conductivity characteristics into different horizontal layered structures. According to the detection results of the above soil resistivity, this paper divides the soil in the calculation area into 7 layers, as shown in table 1.

Table 1. Soil resistivity distribution.

\begin{tabular}{llll}
\hline & Depth $(\mathrm{m})$ & Thickness $(\mathrm{m})$ & Resistivity $(\Omega \cdot \mathrm{m})$ \\
\hline Layer 1 & $0 \sim 100$ & 100 & 6.5 \\
Layer 2 & $100 \sim 500$ & 400 & 120 \\
Layer 3 & $500 \sim 2000$ & 1500 & 455 \\
Layer 4 & $2000 \sim 5000$ & 3000 & 2145 \\
Layer 5 & $5000 \sim 8000$ & 3000 & 5500 \\
Layer 6 & $8000 \sim 10000$ & 2000 & 5100 \\
Layer 7 & $>10000$ & infinite & 8500 \\
\hline
\end{tabular}

Combining the power grid model and the soil model, the DC bias model of Zhejiang Power Grid can be obtained.

\section{DC Bias Calculation Results}

According to the experience of bias control, the DC bias current limits of $500 \mathrm{kV}$, $220 \mathrm{kV}$, and $110 \mathrm{kV}$ substations are $20 \mathrm{~A}, 15 \mathrm{~A}$, and $10 \mathrm{~A}$ respectively $[11,12]$. When Baihetan-Zhejiang HVDC unipolar operating current is $5000 \mathrm{~A}$, the substations with current exceeding the limit near the ground electrode are shown in table 2, based on the simulation model of Zhejiang grid. There are 6 substations with bias current exceeding the limit. The maximum DC bias current is $220 \mathrm{kV}$ Ji' an station, up to $105 \mathrm{~A}$. The main reason is that it is very close to the ground electrode, only $15 \mathrm{~km}$. Other substations are mainly distributed in the nearby Huzhou and Hangzhou areas.

Table 2. Baihetan ground electrode current overrun substation.

\begin{tabular}{|c|c|c|c|c|}
\hline $\begin{array}{l}\text { Voltage } \\
\text { level }\end{array}$ & Substation Transformer type & $\begin{array}{l}\text { High voltage side } \\
\text { (series winding) (A) }\end{array}$ & $\begin{array}{l}\text { Medium voltage side } \\
\text { (common winding) (A) }\end{array}$ & $\begin{array}{l}\text { Distance to DC } \\
\text { ground electrode } \\
(\mathrm{km})\end{array}$ \\
\hline $220 \mathrm{kV}$ & 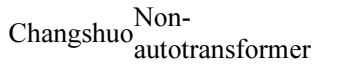 & 37.10 & -32.70 & 26 \\
\hline $220 \mathrm{kV}$ & Ji'an Autotransformer & 73.10 & 105.00 & 14 \\
\hline $220 \mathrm{kV}$ & Autotransformer & -3.79 & -20.40 & 66 \\
\hline $220 \mathrm{kV}$ & Shengshan Autotransformer & 1.09 & -17.30 & 74 \\
\hline $220 \mathrm{kV}$ & $\begin{array}{ll}\text { Yangzi } & \begin{array}{l}\text { Non- } \\
\text { autotransformer }\end{array}\end{array}$ & 17.60 & 18.30 & 39 \\
\hline $110 \mathrm{kV}$ & $\begin{array}{l}\text { Non- } \\
\text { autotransformer }\end{array}$ & 28.6 & 0 & 40 \\
\hline
\end{tabular}

Since the Jinsu ground electrode is located in Jiangsu Province near Huzhou, this HVDC will also cause DC bias effects on substations in Huzhou. Therefore, it is necessary to calculate the DC current of the substation winding of the Huzhou Power Grid when the Jinsu HVDC is unipolar operating. There are 20 substations in Huzhou whose current exceeds the limit. Among them, the substations that overlap with Baihetan-Zhejiang DC are shown in table 3. 
According to the above calculation results, the Baihetan-Zhejiang HVDC has a greater impact on the Huzhou and Hangzhou substations near the ground electrode, it is necessary to provide treatment suggestions for the substations in the above-mentioned areas. At present, there are two main treatment methods: one is resistance limiting, and the other is capacitance blocking. The resistance limiting method is to connect a resistor in series at the neutral point of the transformer to limit the DC current within the range. The capacitor DC blocking method is to connect a capacitor in series with the neutral point of the transformer to completely block the DC path.

Table 3. Jinsu ground electrode current overrun substation.

\begin{tabular}{llllll}
\hline $\begin{array}{l}\text { Voltage } \\
\text { level }\end{array}$ & Substation & Transformer type & $\begin{array}{l}\text { High voltage side } \\
\text { (series winding) }\end{array}$ & $\begin{array}{l}\text { Medium voltage side Distance to DC } \\
\text { (common winding) } \\
\text { (A) }\end{array}$ & $\begin{array}{l}\text { ground electrode } \\
\text { (km) }\end{array}$ \\
\hline $220 \mathrm{kV}$ & Changshuo & Non-autotransformer & -15.70 & -2.98 & 74 \\
$220 \mathrm{kV}$ & Ji'an & Autotransformer & -17.50 & -14.40 & 83 \\
$220 \mathrm{kV}$ & Kunlun & Autotransformer & -3.67 & -28.80 & 63 \\
$220 \mathrm{kV}$ & Shengshan & Autotransformer & -20.00 & 36.20 & 29 \\
$220 \mathrm{kV}$ & Yangzi & Non-autotransformer & -19.50 & -14.20 & 76 \\
\hline
\end{tabular}

Firstly, according to the calculation results of Jinsu HVDC, the substations that need to be treated and observed in the Huzhou area are divided into three priority levels according to risk:

(1) There is only 1 substation whose winding current is positive and exceeds the limit, which is the priority treatment substation.

(2) After the priority substation is treated, the transfer of the DC bias current will cause the current of the adjacent substation to increase, and these two stations with positive currents need to be further treated. Considering the dispersion of the calculated parameters, it is recommended to treat after testing.

(3) Substations with negative currents are not the source of the bias current entering the AC grid, so there is no need for direct control. However, it is necessary to test the substations whose current exceeds the limit before the treatment of (2).

After taking the above-mentioned treatment measures for Jinsu HVDC, the bias current of all substations is controlled within the limit. Among them, Shengshan is the priority substation for governance, and Kunlun are the observation substations.

After Jinsu HVDC treatment, supplementary treatment of Baihetan-Zhejiang HVDC is required. The treatment plan of each substation is shown in table 4.

Table 4. Treatment plan.

\begin{tabular}{|c|c|c|c|c|c|}
\hline $\begin{array}{l}\text { Voltage } \\
\text { level }\end{array}$ & Substation & Transformer type & Winding & Equipment & Priority levels \\
\hline $220 \mathrm{kV}$ & Shengshan & Autotransformer & Common winding & Resistance & $\begin{array}{l}\text { Priority } \\
\text { treatment }\end{array}$ \\
\hline $220 \mathrm{kV}$ & Kunlun & Autotransformer & / & / & Observation \\
\hline $220 \mathrm{kV}$ & Changshuo & Non-autotransformer & $\begin{array}{l}\text { High voltage winding and } \\
\text { Medium voltage winding }\end{array}$ & Resistance & $\begin{array}{l}\text { Supplementary } \\
\text { treatment }\end{array}$ \\
\hline $220 \mathrm{kV}$ & Ji'an & Autotransformer & Common winding & Resistance & $\begin{array}{l}\text { Supplementary } \\
\text { treatment }\end{array}$ \\
\hline $220 \mathrm{kV}$ & Yangzi & Non-autotransformer & $\begin{array}{l}\text { High voltage winding and } \\
\text { Medium voltage winding }\end{array}$ & Resistance & $\begin{array}{l}\text { Supplementary } \\
\text { treatment }\end{array}$ \\
\hline
\end{tabular}

After adopting the above treatment measures, when the Baihetan-Zhejiang HVDC is in unipolar operation, the bias current distribution of the over-limit substation of the 
Huzhou power grid is shown in table 5. After treatment, the bias currents of all substations are within the limit, which reflects the effectiveness of the treatment plan.

Table 5. Calculation result of bias current after treatment.

\begin{tabular}{llllll}
\hline $\begin{array}{l}\text { Voltage } \\
\text { level }\end{array}$ & Substation & Transformer type & $\begin{array}{l}\text { High voltage side } \\
\text { (series winding) } \\
(\mathrm{A})\end{array}$ & $\begin{array}{l}\text { Medium voltage side Distance to DC } \\
\text { (common winding) } \\
(\mathrm{A})\end{array}$ & $\begin{array}{l}\text { Dround electrode } \\
\text { (km) }\end{array}$ \\
\hline $220 \mathrm{kV}$ & Changshuo & Non-autotransformer & 4.26 & 2.77 & 74 \\
$220 \mathrm{kV}$ & Ji'an & autotransformer & 10.70 & 7.11 & 83 \\
$220 \mathrm{kV}$ & Kunlun & autotransformer & -2.12 & -13.40 & 63 \\
$220 \mathrm{kV}$ & Shengshan & autotransformer & 0.60 & -0.32 & 29 \\
$220 \mathrm{kV}$ & Yangzi & non-autotransformer & 1.57 & 1.49 & 76 \\
$1100 \mathrm{kV}$ & Wangjia & non-autotransformer & 5.3 & 0 & 40 \\
\hline
\end{tabular}

\section{Conclusion}

Based on the field-circuit coupling method, this paper establishes a DC bias model for Zhejiang Power Grid, and proposes treatment measures for substations with current overruns in the area. The specific conclusions are as follows:

(1) The up-ground model of the power grid in Zhejiang is established, mainly including substations locations, transformers and connection types, bus, overhead lines and neutral grounding type.

(2) The underground model is mainly the soil model. The resistivity of the soil is calculated by the quadrupole method and the magnetotelluric sounding method, then the earth soil layering model with different resistivity is established.

(3) The bias current caused by the unipolar operation of Baihetan-Zhejiang Jinsu HVDCs is analysed, and effective treatment measures are proposed based on the calculation results. The treatment results show that the proposed plan can effectively restrain the bias current in Zhejiang Power Grid and has engineering practical value.

\section{References}

[1] Zhong L, Lu P, Qiu Z and Cai H 2003 The influence of current of dc earthing electrode on directly grounded transformer High Voltage Engineering 29 (8) 12-14.

[2] Mei G, Liang W, Liu Y and Sun Y 2009 Development of the DC current blocking device in capacitorresistor or transformer High Voltage Engineering 35 (10) 2582-2585.

[3] Ma Z 2007 A potential compensation method to eliminate transformer neutral direct current produced by HVDC Guangdong Electric Power 20 (5) 1-5.

[4] Li C 2005 Effect of DC monopole operation on AC transformers East China Electric Power 33 (1) 3638

[5] Marti L, Rezaei-Zare A and Narang A 2013 Simulation of transformer hotspot heating due to geomagnetically induced currents IEEE Trans. on Power Delivery 28 (1) 320-327.

[6] IEEE Transmission and Distribution Committee Working Group 1993 Geomagnetic disturbance effects on power systems IEEE Trans. on Power Delivery 8 (3) 1206-1216.

[7] Picher P, Boldue L, Dutil A, et al. 1997 Study of the acceptable DC current limit in core-form power transformers IEEE Trans. on Power Delivery 12 (1) 257-265.

[8] Shi M, Wu B, Jin Y, et al. 2018 Research summary on the impacts of DC magnetic bias on transformer High Voltage Apparatus 54 (7) 20-36+43.

[9] Yang N, Chen Y, Pan Z, et al. 2018 Influence prediction and suppression research of DC bias in Eastern Junggar-Southern Anhui $\pm 1100 \mathrm{kV}$ UHVDC receiving-end grid Power System Technology 42 (2) 380-386. 
[10] Ding D, Zhao D, Zhang X, et al. 2016 Investigation of vibration impacts on HVAC transformer from HVDC system under monopole operation IEEE Transactions on Dielectrics and Electrical Insulation 23 (3) 1386-1392.

[11] Ma Y and Yan Z 2018 Influence of DC bias in sending-end gird with multi-HVDC and its countermeasures Smart Power 46 (11) 98-104.

[12] Zhou Y, Quan J, Wen X, et al. 2015 HVDC ground electrode distributed modeling and application Power System Technology 39 (2) 387-392. 